\title{
Epigallocatechin gallate inhibits the growth of human lung cancer by directly targeting the EGFR signaling pathway
}

\author{
YU-CHAO MA ${ }^{1}$, CUI LI ${ }^{3}$, FENG GAO $^{2}$, YAN XU ${ }^{3}$, ZHI-BIN JIANG ${ }^{1}$, JIAN-XIN LIU $^{1}$ and LONG-YU JIN ${ }^{1}$ \\ Departments of ${ }^{1}$ Cardiothoracic Surgery, and ${ }^{2}$ Ultrasound, The Third Xiangya Hospital, Central South University, \\ Changsha 410013; ${ }^{3}$ Key Laboratory of Cancer Proteomics of the Chinese Ministry of Health, \\ Xiangya Hospital, Central South University, Changsha 410008, P.R. China
}

Received October 12, 2013; Accepted December 2, 2013

DOI: $10.3892 /$ or.2013.2933

\begin{abstract}
Epigallocatechin gallate (EGCG), the major biologically active compound in green tea, is a well-known chemoprevention agent. Although several reports have shown that EGCG exerts its anticancer activity by targeting specific cell signaling pathways, the underlying molecular mechanism(s) are only partially understood. In the present study, we report that EGCG had a profound antiproliferative effect on human lung cancer cells. EGCG inhibited anchorageindependent growth and induced cell cycle $\mathrm{G}_{0} / \mathrm{G}_{1}$ phase arrest. The mechanism underlying EGCG antitumor potency was mainly dependent on suppression of the EGFR signaling pathway. Short-term EGCG exposure substantially decreased EGF-induced EGFR, AKT and ERK1/2 activation. Moreover, long-term EGCG treatment not only inhibited total and membranous EGFR expression, but also markedly attenuated EGFR nuclear localization and expression of the downstream target gene cyclin D1, indicating that EGCG treatment suppressed EGFR transactivation. Additionally, knockdown of EGFR in lung cancer cells decreased their sensitivity to EGCG. Thus, inhibition of the EGFR signaling pathway may partly contribute to the anticancer activity of EGCG.
\end{abstract}

\section{Introduction}

Green tea is a popular type of tea worldwide. Epidemiological investigations and experimental studies have shown that green tea has chemopreventive effects for a wide range of malignancies, including lung, stomach, breast, prostate, pancreatic, ovarian, liver, colorectal and skin cancer (1-10). Mechanistic

Correspondence to: Dr Long-Yu Jin, Department of Cardiothoracic Surgery, The Third Xiangya Hospital, Central South University, 138 Tongzipo Road, Yuelu, Changsha 410013, P.R. China

E-mail: limcs51@gmail.com

Abbreviations: EGCG, epigallocatechin gallate; EGFR, epidermal growth factor receptor

Key words: epigallocatechin gallate, epidermal growth factor receptor, lung cancer studies indicate that EGCG, the major component of green tea, appears to be the primary active ingredient responsible for its biological effects. EGCG reportedly exhibits its cancer preventive effects through regulation of multiple signaling pathways, including suppression of various protein kinases $(1,11,12)$, disruption of the activation of transcription factors, and alteration of the expression of genes involved in cell proliferation, angiogenesis and apoptosis, thereby imparting strong cancer chemopreventive as well as therapeutic effects $(13,14)$.

Epidermal growth factor receptor (EGFR) is a transmembrane glycoprotein that possesses intrinsic receptor tyrosine kinase activity. The EGFR signaling pathway is known to play a major role in tumor genesis by regulating cell proliferation, survival and metabolism (15). Upon ligand binding, activated EGFR recruits, phosphorylates and activates a number of important signaling molecules such as PLC- $\gamma$, Ras, PI-3K and JAK2 $(16,17)$. These EGFR downstream signaling cascades are conventional early transient responses and mainly depend on EGFR tyrosine kinase activity (18). Moreover, EGFR and its ligands have repeatedly been observed in the nucleus $(15,19,20)$. Different stimuli such as the EGF ligand or ultraviolet radiation may promote the nuclear translocation of EGFR. Nuclear EGFR interacts with numerous transcription factors such as signal transducer and activator of transcription 3 (STAT3), STAT5 and transcription factor E2F1, and acts as a transcriptional factor to regulate the expression of downstream target genes. Additionally, other DNA-binding partners such as proliferating cell nuclear antigen or DNA protein kinase (DNA-PK) are able to bind with nuclear EGFR and induce PCNA stability or DNA repair (19,21-24). These findings indicate that nuclear EGFR is involved in a number of physiological and pathological processes, such as proliferation, inflammation, metastasis, DNA repair and resistance to DNA-damaging radiation or chemotherapy drugs (15).

Lung cancer is the leading cause of cancer-related death in the world, and non-small cell lung carcinoma (NSCLC) accounts for $80 \%$ of lung cancer cases (25-27). Overexpression and hyperactivation of receptor tyrosine kinases such as EGFR are known to be important in lung cancer carcinogenesis, leading to the development of specific targeted therapies $(28,29)$. Specific receptor antagonists have shown efficacy in clinical practice, yet tumors often become resistant to these therapies due to an EGFR second mutation or other 
tyrosine kinases such as c-Met overexpression, and the 5-year survival for these patients remains poor at $<15 \%$ (28). A major challenge in the treatment of lung cancer is the identification of novel therapeutic targets or the development of new anticancer agents that can supplement current chemotherapy (30).

In the present study, we demonstrated that natural compound EGCG markedly inhibited the growth of lung cancer by directly targeting the EGFR signaling pathway. EGCG not only decreased EGFR tyrosine kinase activity, but also suppressed nuclear localization of EGFR and EGFRmediated expression of downstream target gene cyclin D1.

\section{Materials and methods}

Cell culture and transfection. All cell lines were obtained from the American Type Culture Collection (ATCC; Manassas, VA, USA) and grown in a $37^{\circ} \mathrm{C}$ incubator with $5 \% \mathrm{CO}_{2}$ according to ATCC protocols. For transfection experiments, Lipofectamine $^{\mathrm{TM}} 2000$ transfection reagent (Invitrogen, Carlsbad, CA, USA) was used according to the manufacturer's instructions.

Reagents and antibodies. EGCG was obtained from Sigma (St. Louis, MO, USA). Anti-EGFR, anti-p-EGFR (Tyr1068), anti-AKT (pan), anti-p-AKT (Ser473), anti-ERK1/2, anti-pERK1/2 (Thr202/Tyr204), anti-S6, anti-p-S6 (Ser235/236), anti-cyclin D1, anti- $\alpha$-tubulin and anti-laminB antibodies were purchased from Cell Signaling Technology, Inc. (Danvers, MA, USA). Anti- $\beta$-actin, anti-rabbit IgG-HRP and anti-mouse IgG-HRP were purchased from Santa Cruz Biotechnology (Santa Cruz, CA, USA). Anti-N-cadherin was purchased from BD Biosciences (San Jose, CA, USA).

Western blotting. Cells were harvested by trypsinization and pelleted by centrifugation. Cell pellets were lysed in Nonidet $\mathrm{P}-40$ cell lysis buffer (50 mM Tris- $\mathrm{HCl}, \mathrm{pH} 8.0,150 \mathrm{mM}$ $\mathrm{NaCl}, 0.5 \%$ Nonidet $\mathrm{P}-40$ and protease inhibitor mixture). Protein concentrations were determined by the Bradford assay (Bio-Rad Laboratories, Hercules, CA, USA). Proteins were separated by SDS-PAGE and electrically transferred to polyvinylidene difluoride membranes (Millipore, Billerica, MA, USA). After blocking in 5\% non-fat dry milk in TBS, the membranes were hybridized to specific primary antibodies overnight at $4^{\circ} \mathrm{C}$, washed 3 times with TBS-Tween-20, and then incubated with secondary antibodies conjugated with horseradish peroxidase for $1 \mathrm{~h}$ at room temperature. Next, the membranes were washed 3 times in TBS-Tween-20 at room temperature. The protein bands were visualized using ECL chemiluminescence reagents (Pierce Chemical Co., Rockford, IL, USA) according to the manufacturer's protocol.

Lentiviral infection. Lentivirus plasmids (pLKO.1-shEGFR \#1, TRCN0000039633; pLKO.1-shEGFR \#2, TRCN0000121068) were purchased from Thermo Scientific (Huntsville, AL, USA). We co-transfected $p L K O .1$-shEGFR with PSPAX2 and $P M D 2-G$ into $293 \mathrm{~T}$ cells. Viral supernatant fractions were collected and infected into A549 lung cancer cells with $10 \mu \mathrm{g} / \mathrm{ml}$ Polybrene. After a 24-h infection, the medium was replaced with fresh medium containing the appropriate concentration of puromycin. Appropriate experiments were conducted with these cells until the control cells (without infection) completely died (usually 2-3 days) in the puromycin medium.

Subcellular proteome fractionation. The subcellular proteome fractions were prepared using the ProteoExtract Subcellular Proteome Extraction kit (Millipore) according to the manufacturer's instructions.

Soft agar colony assay. To examine the anchorage-independent growth, lung cancer cells were suspended $(10,000$ cells $/ \mathrm{ml})$ in $1 \mathrm{ml} 0.3 \%$ agar with Eagle's basal medium containing $10 \%$ FBS, $1 \%$ antibiotics, and different concentrations of EGCG $(0$, 10,20 and $40 \mu \mathrm{mol} / \mathrm{l}$ ) overlaid into 6 -well plates containing a $0.6 \%$ agar base. The cultures were maintained in an incubator at $37^{\circ} \mathrm{C}$ with $5 \% \mathrm{CO}_{2}$ for $1-2$ weeks. The colonies were counted under a microscope with the Image-Pro Plus software program (Media Cybernetics, Silver Spring, MD, USA).

Flow cytometry. Flow cytometry was used to quantify cells in each phase of the cell cycle. Cells $\left(2 \times 10^{5}\right)$ were seeded into 6 -well plates and treated with various concentrations of EGCG for $24 \mathrm{~h}$. The cells were harvested and washed with PBS twice and then fixed in $70 \%$ ethanol overnight at $4^{\circ} \mathrm{C}$. The cells were counterstained in the dark with $50 \mu \mathrm{g} / \mathrm{ml}$ phosphatidyl inositol and $0.1 \%$ ribonuclease A (RNase A) in $400 \mu 1 \mathrm{PBS}$ at $25^{\circ} \mathrm{C}$ for $30 \mathrm{~min}$. The stained cells were assayed and quantified by a FACSort flow cytometer (BD Biosciences).

Statistical analysis. All statistical analyses were performed with SPSS software (version 13.0). The experiments were performed in triplicate. All quantitative data are expressed as mean values \pm standard deviation. The significant differences between two groups were assessed by a 2-tailed Student's t-test. A probability value $(\mathrm{P})<0.05$ was considered to represent a statistically significant difference.

\section{Results}

EGCG inhibits the anchorage-independent growth of human lung cancer cells. In the present study, we first examined the effect of EGCG on the anchorage-independent growth of human lung cancer cell lines, A549, H1650 and H460. EGCG significantly inhibited A549 cell growth in soft agar in a dosedependent manner (Fig. 1A). At $20 \mu \mathrm{M}$, EGCG substantially inhibited the colony formation of A549 cells in soft agar, and the inhibition rate reached $80 \%$ at the concentration of $40 \mu \mathrm{M}$. Meanwhile, we also investigated the effect of EGCG on the growth of 2 other lung cancer cell lines H1650 and H460. EGCG dose-dependently inhibited the growth of these cell lines on soft agar. At a concentration of $20 \mu \mathrm{M}$, the inhibition rate reached 60 and $30 \%$, respectively (Fig. $1 \mathrm{~B}$ and C). EGCG dose-dependently inhibited the growth of lung cancer cells in soft agar.

Short-term exposure to EGCG inhibits EGF-induced EGFR phosphorylation and its downstream signaling pathway. Previous studies have reported that the EGFR signaling pathway is often deregulated in human lung cancer and plays a dominant role in the proliferation and survival of cancer 
A
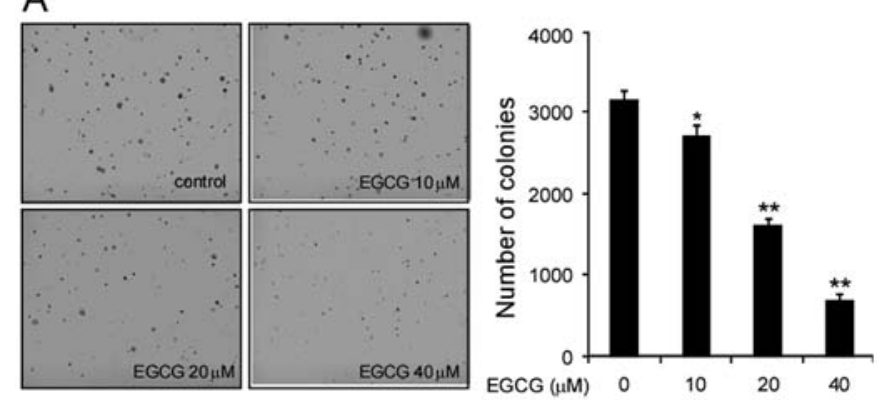

B
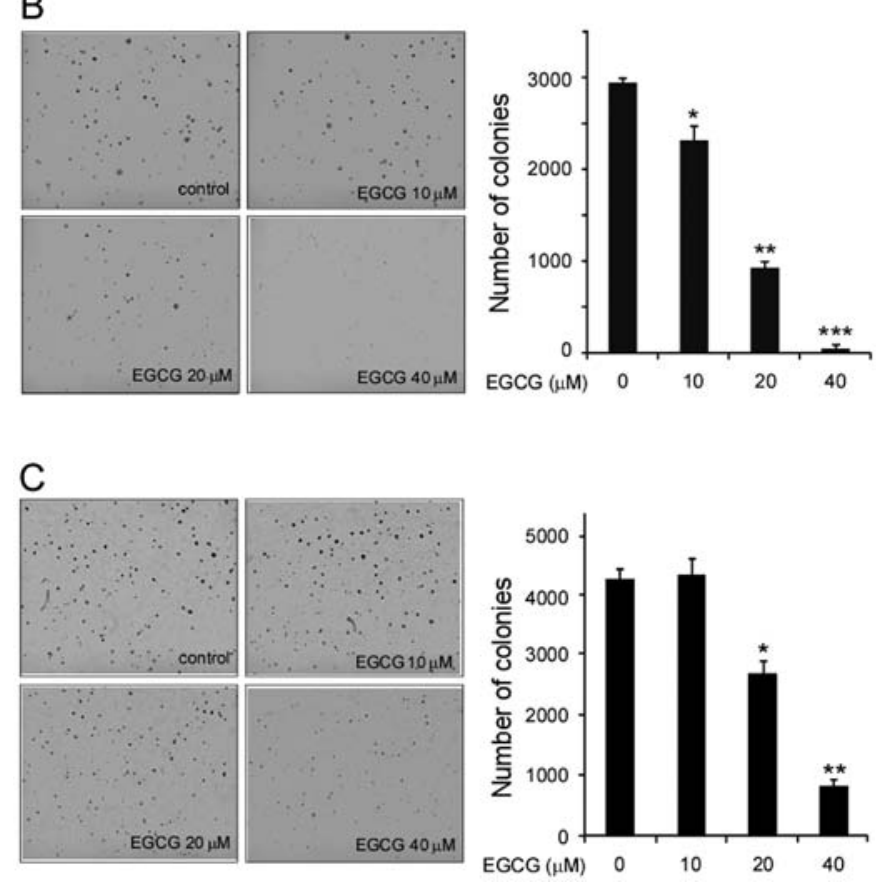

Figure 1. Inhibitory effect of EGCG on anchorage-independent growth of human lung cancer cells. EGCG inhibited anchorage-independent growth in a panel of human lung cancer cell lines: (A) A549, (B) H1650 and (C) H460. A colony formation assay was performed as described in Materials and methods. The data represent the colony formation ability of human lung cancer cells treated with different concentrations of EGCG compared with the dimethyl sulfoxide-treated group. The average colony number was calculated from 3 separate experiments. Columns, mean value of the number of colonies as determined from the 3 independent experiments; bars, standard deviation. ${ }^{*} \mathrm{P}<0.05,{ }^{* *} \mathrm{P}<0.01,{ }^{* * *} \mathrm{P}<0.001$ indicate significant suppression of colony formation by EGCG.

cells. Therefore, we investigated the effect of EGCG on the EGFR signaling pathway. Treatment of A549 cells with EGCG significantly inhibited EGFR activation. Although a low concentration $(10 \mu \mathrm{M})$ of EGCG had little effect on EGFR phosphorylation, the EGF-induced EGFR activation was inhibited $>50 \%$ in the A549 cells in a dose-dependent manner following treatment with 20 to $40 \mu \mathrm{M}$ EGCG. EGCG suppressed the activation of EGFR downstream kinases, such as Akt, ERK1/2 and S6 in the A549 cells (Fig. 2A). To further confirm the effect of EGCG on EGFR phosphorylation, we determined EGF-induced EGFR phosphorylation at various time-points. Consistent with the above results, EGCG suppressed EGFR activation as well as its downstream kinases Akt and ERK1/2 at all time points (15,30 and $60 \mathrm{~min})$
A

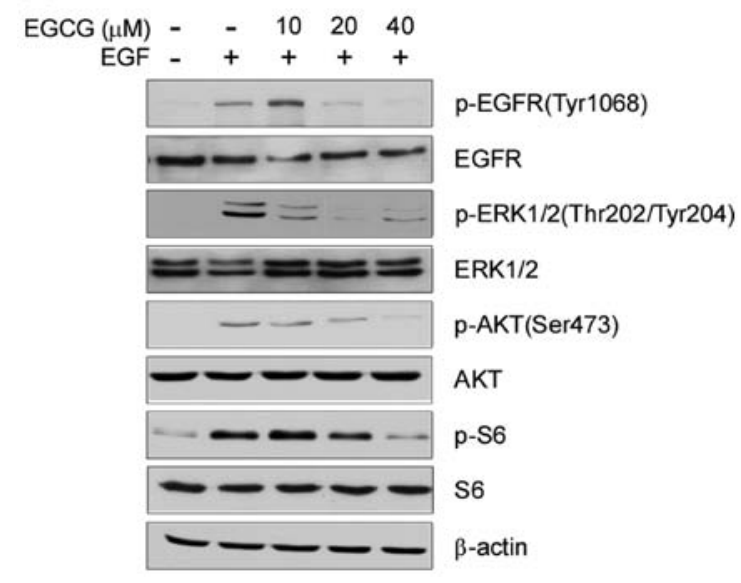

B

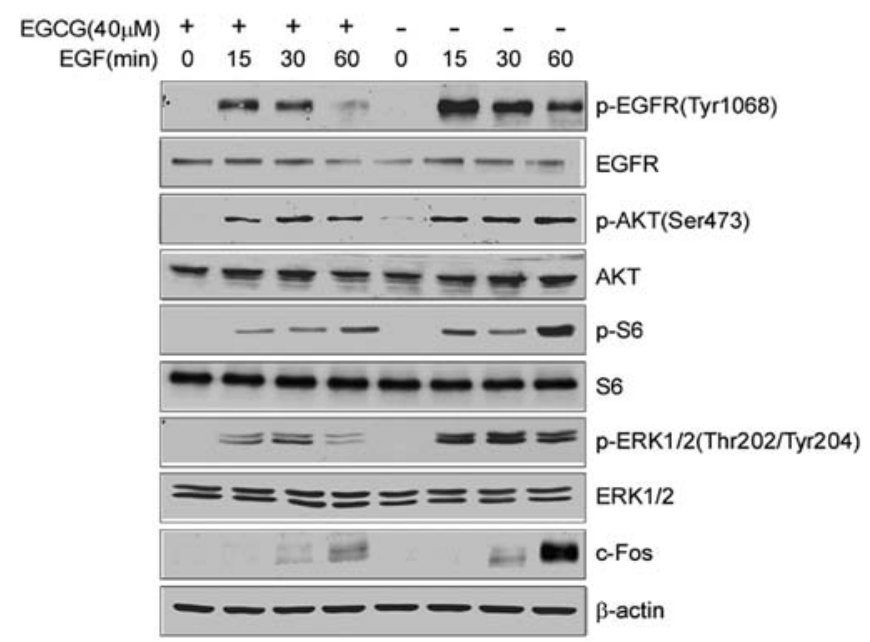

Figure 2. EGCG affects EGF-induced epidermal growth factor receptor signaling in A549 lung cancer cells. (A) EGCG dose-dependently inhibited the EGF-induced EGFR signaling pathway. A549 cells were starved in a serum-free medium for $24 \mathrm{~h}$, and then treated with EGCG at the indicated concentrations for $2 \mathrm{~h}$. After stimulation with EGF (20 ng/ml) for $30 \mathrm{~min}$, the cells were harvested and protein levels were determined by western blotting. (B) EGCG time-dependently inhibited the EGF-induced EGFR signaling pathway. A5 49 cells were starved in a serum-free medium for $24 \mathrm{~h}$. Following pre-treatment with $40 \mu \mathrm{mol}$ EGCG for $2 \mathrm{~h}$, the cells were stimulated with EGF $(20 \mathrm{ng} / \mathrm{ml})$ for various times $(15,30$ and $60 \mathrm{~min})$, and the cell lysates were harvested and protein levels were determined by western blotting.

(Fig. 2B). As c-Fos is one of the most important immediate early genes regulated by EGFR to initiate downstream target gene transcription upon growth factor stimulation, EGCG may regulate c-Fos expression. As expected, together with the suppression of phosphorylation of EGFR, EGF-induced c-Fos expression was inhibited by EGCG treatment (Fig. 2B), suggesting that EGCG decreases EGF-induced EGFR activation and signaling transduction.

Long-term exposure to EGCG decreases the expression levels of EGFR in human lung cancer cells. EGF-induced phosphorylation and activation of the EGFR signaling pathway were inhibited after transient EGCG treatment, indicating that EGCG dose-dependently inhibited EGFR tyrosine kinase activity. Then, we investigated the effect of EGCG on EGFR 

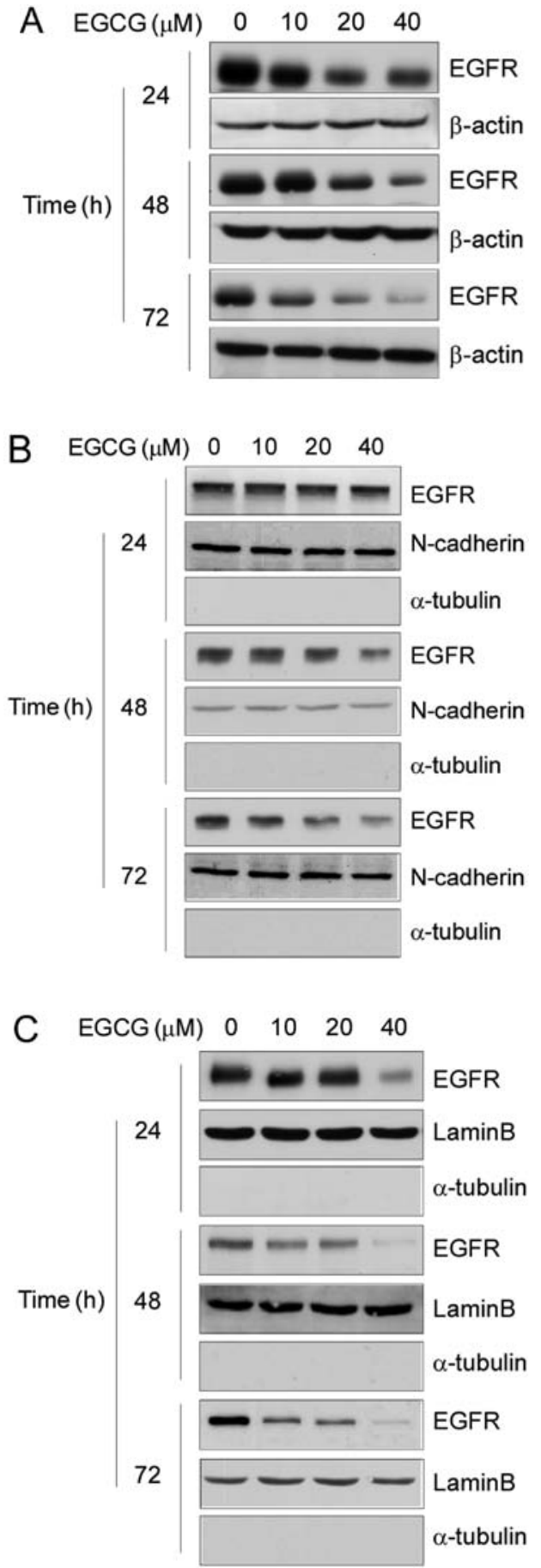

Figure 3. EGCG affects the expression levels of EGFR in A549 cells (A) EGCG suppressed EGFR expression in A549 cells. A549 cells were treated with different concentrations of EGCG for various time-points as indicated, the whole cell lysates were harvested and protein levels were determined by western blotting. EGCG treatment (B) decreased the expression level of membrane-associated EGFR and (C) inhibited EGFR nuclear localization. A549 cells were treated with different concentrations of EGCG for various time-points as indicated. Subsequently, the membranous and nuclear fractions were isolated as described in Materials and methods and analyzed by western blotting using the indicated antibodies.

expression after long-term exposure. As shown in Fig. 3A, long-term exposure to EGCG significantly suppressed the expression of EGFR in a dose-dependent and time-dependent manner. Following treatment with EGCG at $20 \mu \mathrm{M}$ for $48 \mathrm{~h}$,
A
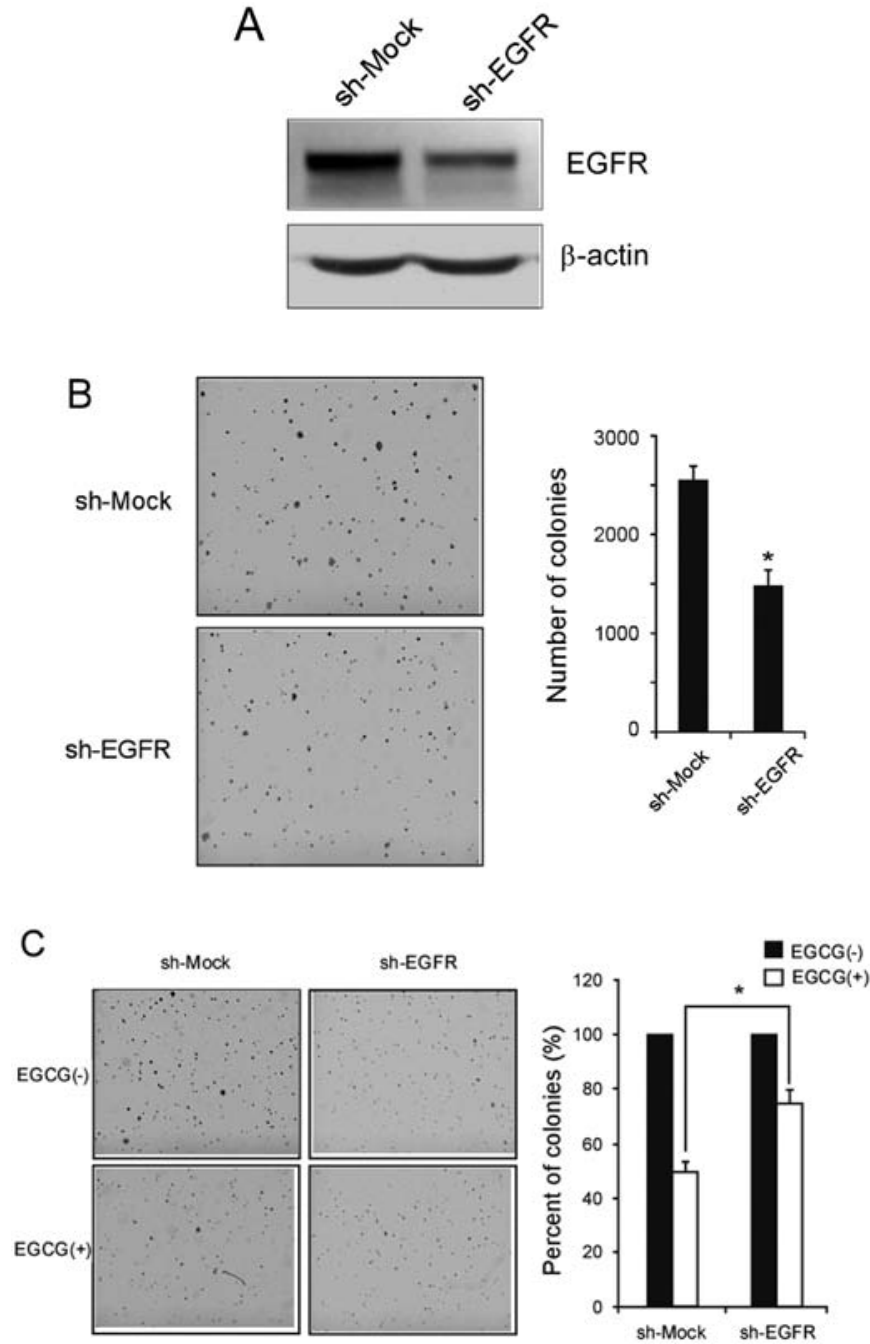

Figure 4. Knockdown of EGFR in A549 cells decreases the sensitivity to EGCG. (A) The efficiency of EGFR shRNA in A549 cells was detected by western blotting. (B) Knockdown of EGFR attenuated the anchorage-independent growth of A549 cells. (C) Sensitivity of A549 cells transfected with mock shRNA (sh-Mock) or EGFR shRNA (sh-EGFR) to EGCG treatment. Stable knockdown of EGFR in A549 cells by lentiviral infection and soft agar colony formation assays were performed as described in Materials and methods. Representative images (B and C, left panels) are shown. The graphs (B and $\mathrm{C}$, right panels) show data from at least 3 independent experiments expressed as means $\pm \mathrm{SD} .{ }^{*} \mathrm{P}<0.05$ (Student's t-test) indicates a significant decrease in colony formation of EGFR-knockdown cells (B) and significant decrease in sensitivity to EGCG of EGFR-knockdown A549 cells (C).

the total EGFR protein level was markedly decreased. Next, we purified the subcellular fractions to determine whether EGCG treatment regulates EGFR expression both on the cellular membrane and in the nucleus. As shown in Fig. 3B, in A549 lung cancer cells, the membranous expression level of EGFR exhibited no obvious decrease following treatment with $40 \mu \mathrm{M}$ EGCG for $24 \mathrm{~h}$; however, following a 72-h EGCG treatment, the EGFR membranous expression level was significantly reduced. Moreover, EGCG had a high inhibitory effect on nuclear EGFR expression, following treatment with $40 \mu \mathrm{M}$ EGCG for $24 \mathrm{~h}$ (Fig. 3C). The expression of EGFR was markedly decreased at $72 \mathrm{~h}$, and the expression of EGFR was almost completely suppressed, indicating that long-term EGCG treatment inhibited the EGFR expression both on the cellular membrane and in the nucleus. 

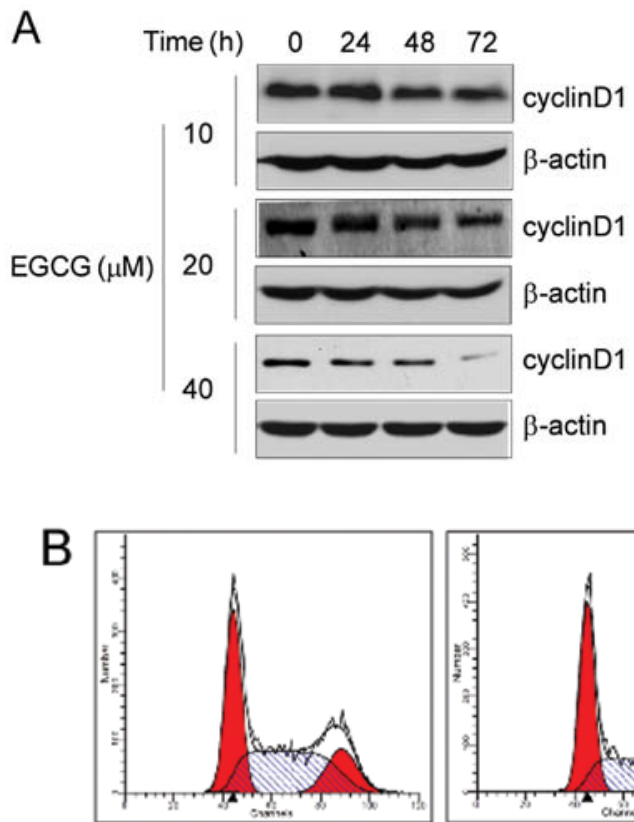

EGCG: $0 \mu \mathrm{M}$

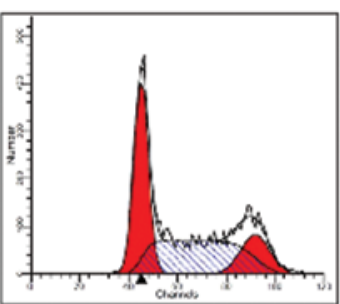

EGCG: $10 \mu \mathrm{M}$
Figure 5. EGCG regulates cyclin D1 expression and cell cycle progression in A549 lung cancer cells. (A) EGCG dose-dependently and time-dependently inhibited cyclin D1 expression in A549 cells. A549 cells were treated with different concentrations of EGCG at various time-points as indicated. The whole cell lysates were harvested and subjected to western blotting. (B) EGCG treatment dose-dependently induced cell cycle arrest at the $\mathrm{G}_{0} /$ $\mathrm{G}_{1}$ phase. Equal numbers of A549 cells in 6-well plates were treated with various concentrations of EGCG or without EGCG for $24 \mathrm{~h}$, and cell cycle analysis was carried out by flow cytometry. The data are presented from 3 independent experiments expressed as means $\pm \mathrm{SD}$. ${ }^{*} \mathrm{P}<0.05$ (Student's t test) indicates a significant increase in the number of A549 cells in the $G_{0} / G_{1}$ phase following EGCG treatment.

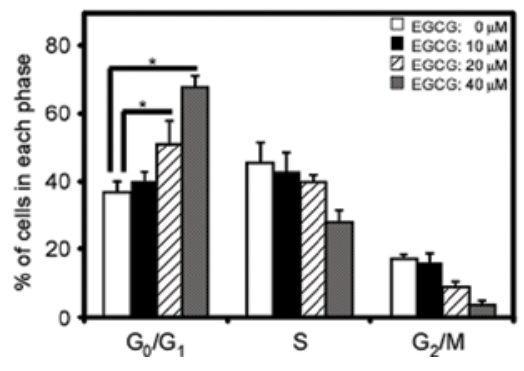

Knockdown of EGFR reduces the sensitivity of lung cancer cells to EGCG. We examined whether the knockdown of EGFR expression influences the sensitivity of A549 lung cancer cells to EGCG. First, we determined the efficiency of shRNA knockdown, as well as the effect of shRNA transfection on anchorage-independent growth. The expression of EGFR was obviously decreased after shRNA transfection (Fig. 4A). Moreover, the growth of cells in soft agar decreased by $>35 \%$ following transfection when compared with the mock group (Fig. 4B). Next, A549 cells transfected with sh-EGFR or sh-Mock control were treated with EGCG or vehicle and subjected to a soft agar assay. EGCG $(20 \mu \mathrm{M})$ inhibited the anchorage-independent growth of A549 sh-Mock cells by $\sim 50 \%$. In contrast, the inhibition was $25 \%$ in A549 sh-EGFR cells, indicating that A549 cells transfected with EGFR shRNA were resistant to EGCG treatment (Fig. 4C). These results imply that EGFR plays an important role in the sensitivity of A549 cells to the antiproliferative effect of EGCG.

EGCG treatment suppresses cyclin D1 expression and induces cell cycle $G_{0} / G_{I}$ arrest. Previous studies demonstrated that cyclin D1 is an important target gene for nuclear EGFR and is involved in cell cycle regulation $(31,32)$. Based on the above results, we further investigated the effect of EGCG on cyclin D1 expression as well as on cell cycle progression. Long-term treatment of EGCG resulted in downregulation of cyclin D1 expression in A549 lung cancer cells. Following treatment with

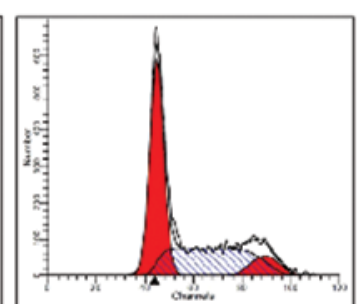

EGCG: $20 \mu \mathrm{M}$

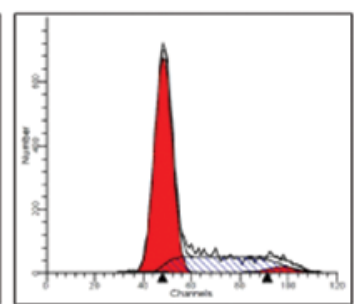

EGCG: $40 \mu \mathrm{M}$
$20 \mu \mathrm{M}$ EGCG for $48 \mathrm{~h}$, the expression of cyclin D1 protein was substantially suppressed (Fig. 5A). Together with the inhibition of cyclin D1, EGCG treatment was accompanied by cell cycle arrest at the $G_{0} / G_{1}$ phase in a concentration-dependent manner. As shown in Fig. 5B, the percentage of cells in the $G_{0} / G_{1}$ phase was increased to $\sim 25 \%$ following treatment with $40 \mu \mathrm{M}$ EGCG. These data indicate that EGCG exerts its antitumor activity via inhibition of EGFR transactivation ability and regulation of expression of its target genes.

\section{Discussion}

The natural compound EGCG is the major polyphenol component in green tea. EGCG is a highly active and promising therapeutic and chemopreventive agent. However, the underlying mechanism for its anticancer activity has not yet been elucidated. The present study identified EGFR as a direct target of EGCG in human lung cancer.

EGFR regulates important tumorigenic processes, including proliferation, apoptosis, angiogenesis and invasion, and along with its ligands, it is frequently overexpressed or hyperactivated during the development and progression of human lung cancer $(28,33)$. Previous studies have revealed that EGCG inhibits the activation of the EGFR and downstream signaling pathways in several types of cancer cells (34-37). Moreover, EGCG suppresses growth factor receptor signaling in human non-small cell lung cancer cells and potentiates the 
antiproliferative activity of c-Met and EGFR inhibitors (38). In the present study, we first examined the antitumor effects of EGCG in several human lung cancer cell lines. EGCG inhibited the anchorage-independent growth of human lung cancer cells in a dose-dependent manner (Fig. 1). Western blotting demonstrated that the EGFR signaling pathway appeared to play an important role in EGCG-mediated lung cancer cell growth inhibition. EGF-induced EGFR activation was markedly decreased by EGCG pre-treatment (Fig. 2). Our findings corroborated previous studies which demonstrated that the effect of EGCG against lung cancer may depend on immediate EGFR activity suppression.

Receptor downregulation is the most prominent regulator of EGFR signal attenuation and involves the internalization and subsequent degradation of the activated receptor in lysosomes. Previous reports demonstrated that EGCG-induced EGFR signaling reduction was partly regulated by $\mathrm{p} 38$-mediated EGFR phosphorylation and internalization in colon cancer $(39,40)$. We then assessed EGFR expression after longterm EGCG exposure in A549 cells. EGCG not only decreased the total EGFR protein levels (Fig. 3A), but also inhibited the EGFR expression on the cell membrane (Fig. 3B). More recent compelling evidence further indicates that EGFR function depends on its subcellular location. EGFR can be shuttled into the cell nucleus and the mitochondrion upon ligand binding, radiation, EGFR-targeted therapy and other stimuli. Nuclear EGFR serves as a transcriptional regulator, tyrosine kinase and mediator of other physiological processes (15). In the present study, we demonstrated for the first time that EGCG markedly inhibited EGFR nuclear localization in a dose- and time-dependent manner (Fig. 3C). Based on these results, we hypothesized that EGCG attenuates EGFR transcription activity. Cyclin D1 is one of the well-known downstream target genes of EGFR. We determined the effect of EGCG on cyclin D1 expression by western blot analysis. Consistent with our hypothesis, the results clearly showed that EGCG potently suppressed cyclin D1 expression in a concentrationdependent manner (Fig. 5A). Additionally, EGCG-mediated cyclin D1 inhibition was accompanied by cell cycle arrest at the $G_{0} / G_{1}$ phase (Fig. 5B). Moreover, knockdown of EGFR expression suppressed A549 cell colony formation in soft agar and decreased the sensitivity of A549 cells to EGCG treatment (Fig. 4). These data provide strong evidence that long-term EGCG exposure markedly downregulates EGFR protein expression as well as its transactivation.

Taken together, EGFR, as a potential and important target of EGCG, offers useful evidence for the rational use and combination treatment of EGCG in lung cancer therapy. We cannot exclude the possibility that EGCG treatment, in addition to decreasing EGFR kinase activity and protein expression, may have antitumor activity by affecting other pathways. Numerous preclinical studies and clinical trials of EGCG are still ongoing in different countries (1), and more research must be conducted to provide further valuable evidence to guide the clinical use of EGCG in lung cancer prevention and therapy.

\section{Acknowledgements}

The present study was supported by the Nature Scientific Foundation of China (grant no. 81371690), the Nature Scientific
Foundation of Hunan Province (grant no. 08JJ6010), the Research Program of the Science and Technology Department of Hunan Province (grant no. 2012FJ4076) and the Research Program of the Science and Technology Department of Hunan Province (grant no. 2012TT2011). We would like to thank Professor Jing Zhou for his excellent English language editing of the manuscript.

\section{References}

1. Kanwar J, Taskeen M, Mohammad I, Huo C, Chan TH and Dou QP: Recent advances on tea polyphenols. Front Biosci (Elite Ed) 4: 111-131, 2012.

2. Jin L, Li C, Xu Y, Wang L, Liu J, Wang D, et al: Epigallocatechin gallate promotes p53 accumulation and activity via the inhibition of MDM2-mediated p53 ubiquitination in human lung cancer cells. Oncol Rep 29: 1983-1990, 2013.

3. Park JS, Khoi PN, Joo YE, Lee YH, Lang SA, Stoeltzing O, et al: EGCG inhibits recepteur d'origine nantais expression by suppressing Egr-1 in gastric cancer cells. Int J Oncol 42: 1120-1126, 2013.

4. Yiannakopoulou EC: Effect of green tea catechins on breast carcinogenesis: a systematic review of in-vitro and in-vivo experimental studies. Eur J Cancer Prev: Aug 9, 2013 (Epub ahead of print).

5. Hagen RM, Chedea VS, Mintoff CP, Bowler E, Morse HR and Ladomery MR: Epigallocatechin-3-gallate promotes apoptosis and expression of the caspase 9a splice variant in $\mathrm{PC} 3$ prostate cancer cells. Int J Oncol 43: 194-200, 2013.

6. Kim SO and Kim MR: (-)-Epigallocatechin 3-gallate inhibits invasion by inducing the expression of Raf kinase inhibitor protein in AsPC1 human pancreatic adenocarcinoma cells through the modulation of histone deacetylase activity. Int $\mathrm{J}$ Oncol 42: 349-358, 2013.

7. Chen H, Landen CN, Li Y, Alvarez RD and Tollefsbol TO: Enhancement of cisplatin-mediated apoptosis in ovarian cancer cells through potentiating G2/M arrest and p21 upregulation by combinatorial epigallocatechin gallate and sulforaphane. J Oncol 2013: 872957, 2013.

8. Kochi T, Shimizu M, Terakura D, Baba A, Ohno T, Kubota M, et al: Non-alcoholic steatohepatitis and preneoplastic lesions develop in the liver of obese and hypertensive rats: suppressing effects of EGCG on the development of liver lesions. Cancer Lett 42: 60-69, 2014.

9. Ogawa K, Hara T, Shimizu M, Nagano J, Ohno T, Hoshi M, et al: (-)-Epigallocatechin gallate inhibits the expression of indoleamine 2,3-dioxygenase in human colorectal cancer cells. Oncol Lett 4: 546-550, 2012.

10. Chiou YS, Sang S, Cheng KH, Ho CT, Wang YJ and Pan MH: Peracetylated (-)-epigallocatechin-3-gallate (AcEGCG) potently prevents skin carcinogenesis by suppressing the PKD1-dependent signaling pathway in $\mathrm{CD} 34^{+}$skin stem cells and skin tumors. Carcinogenesis 34: 1315-1322, 2013.

11. Shimizu M, Adachi S, Masuda M, Kozawa $\mathrm{O}$ and Moriwaki $\mathrm{H}$ : Cancer chemoprevention with green tea catechins by targeting receptor tyrosine kinases. Mol Nutr Food Res 55: 832-843, 2011.

12. Fujiki $\mathrm{H}$ and Suganuma M: Green tea: an effective synergist with anticancer drugs for tertiary cancer prevention. Cancer Lett 324: 119-125, 2012.

13. Surh YJ: Cancer chemoprevention with dietary phytochemicals. Nat Rev Cancer 3: 768-780, 2003.

14. Singh BN, Shankar S and Srivastava RK: Green tea catechin, epigallocatechin-3-gallate (EGCG): mechanisms, perspectives and clinical applications. Biochem Pharmacol 82: 1807-1821, 2011.

15. Han W and Lo HW: Landscape of EGFR signaling network in human cancers: biology and therapeutic response in relation to receptor subcellular locations. Cancer Lett 318: 124-134, 2012.

16. Tebbutt N, Pedersen MW and Johns TG: Targeting the ERBB family in cancer: couples therapy. Nat Rev Cancer 13: 663-673, 2013.

17. Kolch W and Pitt A: Functional proteomics to dissect tyrosine kinase signalling pathways in cancer. Nat Rev Cancer 10: 618-629, 2010. 
18. Avraham R and Yarden Y: Feedback regulation of EGFR signalling: decision making by early and delayed loops. Nat Rev Mol Cell Biol 12: 104-117, 2011.

19. Marti U, Burwen SJ, Wells A, Barker ME, Huling S, Feren AM, et al: Localization of epidermal growth factor receptor in hepatocyte nuclei. Hepatology 13: 15-20, 1991.

20. Brand TM, Iida M, Luthar N, Starr MM, Huppert EJ and Wheeler DL: Nuclear EGFR as a molecular target in cancer. Radiother Oncol 108: 370-377, 2013.

21. Lo HW, Hsu SC, Ali-Seyed M, Gunduz M, Xia W, Wei Y, et al: Nuclear interaction of EGFR and STAT3 in the activation of the iNOS/NO pathway. Cancer Cell 7: 575-589, 2005.

22. Hanada N, Lo HW, Day CP, Pan Y, Nakajima Y and Hung MC: Co-regulation of B-Myb expression by E2F1 and EGF receptor. Mol Carcinog 45: 10-17, 2006.

23. Hung LY, Tseng JT, Lee YC, Xia W, Wang YN, Wu ML, et al: Nuclear epidermal growth factor receptor (EGFR) interacts with signal transducer and activator of transcription 5 (STAT5) in activating Aurora-A gene expression. Nucleic Acids Res 36: 4337-4351, 2008

24. Dittmann K, Mayer C, Fehrenbacher B, Schaller M, Raju U, Milas L, et al: Radiation-induced epidermal growth factor receptor nuclear import is linked to activation of DNA-dependent protein kinase. J Biol Chem 280: 31182-31189, 2005.

25. Siegel R, Naishadham D and Jemal A: Cancer statistics, 2012. CA Cancer J Clin 62: 10-29, 2012.

26. Katlic MR, Facktor MA, Berry SA, McKinley KE, Bothe A Jr and Steele GD Jr: ProvenCare lung cancer: a multi-institutional improvement collaborative. CA Cancer J Clin 61: 382-396, 2011.

27. Ramalingam SS, Owonikoko TK and Khuri FR: Lung cancer: new biological insights and recent therapeutic advances. CA Cancer J Clin 61: 91-112, 2011.

28. Herbst RS, Heymach JV and Lippman SM: Lung cancer. N Engl J Med 359: 1367-1380, 2008.

29. Herbst RS, Fukuoka M and Baselga J: Gefitinib - a novel targeted approach to treating cancer. Nat Rev Cancer 4: 956-965, 2004.

30. Janku F, Stewart DJ and Kurzrock R: Targeted therapy in nonsmall-cell lung cancer - is it becoming a reality? Nat Rev Clin Oncol 7: 401-414, 2010.

31. Tao Y, Song X, Deng X, Xie D, Lee LM, Liu Y, et al: Nuclear accumulation of epidermal growth factor receptor and acceleration of G1/S stage by Epstein-Barr-encoded oncoprotein latent membrane protein 1. Exp Cell Res 303: 240-251, 2005.
32. Shi Y, Tao Y, Jiang Y, Xu Y, Yan B, Chen X, et al: Nuclear epidermal growth factor receptor interacts with transcriptional intermediary factor 2 to activate cyclin D1 gene expression triggered by the oncoprotein latent membrane protein 1 . Carcinogenesis 33: 1468-1478, 2012.

33. Sato M, Shames DS, Gazdar AF and Minna JD: A translational view of the molecular pathogenesis of lung cancer. $J$ Thorac Oncol 2: 327-343, 2007.

34. Shimizu M, Shirakami Y and Moriwaki H: Targeting receptor tyrosine kinases for chemoprevention by green tea catechin, EGCG. Int J Mol Sci 9: 1034-1049, 2008.

35. Masuda M, Suzui M and Weinstein IB: Effects of epigallocatechin-3-gallate on growth, epidermal growth factor receptor signaling pathways, gene expression, and chemosensitivity in human head and neck squamous cell carcinoma cell lines. Clin Cancer Res 7: 4220-4229, 2001.

36. Hou Z, Sang S, You H, Lee MJ,Hong J, Chin KV, et al: Mechanism of action of (-)-epigallocatechin-3-gallate: auto-oxidation-dependent inactivation of epidermal growth factor receptor and direct effects on growth inhibition in human esophageal cancer KYSE 150 cells. Cancer Res 65: 8049-8056, 2005.

37. Adachi S, Nagao T, Ingolfsson HI, Maxfield FR, Andersen OS, Kopelovich L, et al: The inhibitory effect of (-)-epigallocatechin gallate on activation of the epidermal growth factor receptor is associated with altered lipid order in HT29 colon cancer cells Cancer Res 67: 6493-6501, 2007.

38. Milligan SA, Burke P, Coleman DT, Bigelow RL, Steffan JJ, Carroll JL, et al: The green tea polyphenol EGCG potentiates the antiproliferative activity of c-Met and epidermal growth factor receptor inhibitors in non-small cell lung cancer cells. Clin Cancer Res 15: 4885-4894, 2009.

39. Adachi S, Shimizu M, Shirakami Y, Yamauchi J, Natsume H, Matsushima-Nishiwaki R, et al: (-)-Epigallocatechin gallate downregulates EGF receptor via phosphorylation at Ser1046/1047 by p38 MAPK in colon cancer cells. Carcinogenesis 30 : 1544-1552, 2009.

40. Arteaga CL: Epidermal growth factor receptor dependence in human tumors: more than just expression? Oncologist 7 (Suppl 4): S31-S39, 2002. 